\title{
Subretinal versus intravitreal administration of human CD34+ bone marrow-derived stem cells in a rat model of inherited retinal degeneration
}

\author{
Un Chul Park ${ }^{1,2 \#}$, Susanna S. Park ${ }^{3 \#}$, Bo Hee Kim ${ }^{1,2}$, Sung Wook Park ${ }^{1,2}$, Young Joo Kim², Whitney Cary ${ }^{4}$, \\ Johnathon D. Anderson ${ }^{4,5}$, Jan A. Nolta ${ }^{4}$, Hyeong Gon Yu ${ }^{1,2,6}$ \\ ${ }^{1}$ Department of Ophthalmology, College of Medicine, Seoul National University, Seoul, Korea; ${ }^{2}$ Retinal Degeneration Research Laboratory, \\ Seoul National University Hospital Biomedical Research Institute, Seoul, Korea; ${ }^{3}$ Department of Ophthalmology \& Vision Science, University of \\ California Davis. Sacramento, CA, USA; ${ }^{4}$ Stem Cell Program, Institute for Regenerative Cures, University of California Davis, Sacramento, CA, \\ USA; ${ }^{5}$ Department of Otolaryngology, School of Medicine, University of California Davis. Sacramento, CA, USA; ${ }^{6}$ Seoul National University \\ College of Medicine, Interdisciplinary Programs: Stem Cell Biology, Seoul, Korea \\ Contributions: (I) Conception and design: SS Park, HG Yu; (II) Administrative support: YJ Kim, JA Nolta; (III) Provision of study materials or \\ patients: HG Yu, JD Anderson, W Cary; (IV) Collection and assembly of data: UC Park, BH Kim, SW Park; (V) Data analysis and interpretation: \\ UC Park; (VI) Manuscript writing: All authors; (VII) Final approval of manuscript: All authors. \\ \#These authors contributed equally to this work. \\ Correspondence to: Hyeong Gon Yu, MD, PhD. Department of Ophthalmology, Seoul National University College of Medicine, 103 Daehak-ro, \\ Jongno-gu, Seoul 110-799, Korea. Email: hgonyu@snu.ac.kr.
}

Background: To evaluate whether subretinal or intravitreal injection of human CD34+ bone marrowderived stem cells (BMSC) can have protective effects on retinal degeneration that may be enhanced by coadministration of exosomes harvested from human bone marrow mesenchymal stem cells (MSCs).

Methods: Human CD34+ cells were harvested from the mononuclear cell fraction of bone marrow using magnetic beads and labeled with EGFP. Exosomes were harvested from cultured human MSCs under hypoxic conditions. Royal College of Surgeons (RCS) 3-weeks-old rats, immunosuppressed with cyclosporine A, received subretinal or intravitreal injection of CD34+ cells (50,000 cells), CD34+ cells with exosomes $(50,000$ cells $+10 \mu \mathrm{g})$, exosomes alone $(10 \mu \mathrm{g})$, or PBS. Retinal function was examined using electroretinography (ERG), and the eyes were harvested for histologic and immunohistochemical analysis.

Results: The b-wave amplitude of ERG at 2 weeks after injection was significantly higher in eyes with subretinal or intravitreal CD34+ BMSC alone or in combination with exosomes when compared to PBS injected eyes or untreated contralateral eyes. At 4 weeks after injection, the ERG signal decreased in all groups but eyes with subretinal CD34+ BMSCs alone or combined with exosomes showed partially preserved ERG signal and preservation of the outer nuclear layer of the retina near the injection site on histology when compared to eyes with PBS injection. Immunohistochemical analysis identified the human cells in the outer retina. Subretinal or intravitreal exosome injection had no effect on retinal degeneration when administered alone or in combination with CD34+ cells.

Conclusions: Both subretinal and intravitreal injection of human CD34+ BMSCs can provide functional rescue of degenerating retina, although the effects were attenuated over time in this rat model. Regional preservation of the outer retina can occur near the subretinal injection site of CD34+ cells. These results suggest that CD34+ cells may have therapeutic potential in retinal degeneration.

Keywords: CD34; exosome; intravitreal injection; retinal degeneration; subretinal injection

Submitted Jun 15, 2020. Accepted for publication Sep 28, 2020.

doi: $10.21037 /$ atm-20-4662

View this article at: https://dx.doi.org/10.21037/atm-20-4662 


\section{Introduction}

Retinal degenerative diseases, such as inherited retinal diseases and age-related macular degeneration (AMD), are leading causes of incurable blindness worldwide $(1,2)$. Both the inherited and acquired forms of retinal degeneration are characterized by the loss of retinal pigment epithelium (RPE) and photoreceptors. These conditions are progressive since mammalian retina lacks endogenous selfrepair mechanisms to repair the damaged retina (3).

Cellular therapy is a therapeutic option being explored to reverse or limit vision loss associated with retinal degenerative conditions. Cell replacement strategies are being explored in early phase clinical trials using subretinal administration of retinal or RPE cells derived from human embryonic stem cells or induced pluripotent stem cells. These studies show feasibility of the cell administration but efficacy signals are not consistently observed (4-8). In addition, these approaches have limitations, including ethical issues, a shortage of cell sources, immune rejection, and teratogenicity $(9,10)$. An alternative strategy using cellular therapy is optimizing the known paracrine trophic effects of some stem cells, e.g., adult bone marrow-derived stem cells (BMSCs).

Bone marrow is a rich source of adult stem cells. They include hematopoietic stem cells, characterized by the CD34+ cell surface marker in human, and mesenchymal stem cells (MSCs) that can be harvested and expanded in culture. Both types of BMSCs have known paracrine repair effects on damaged tissue and are mobilized in response to tissue injury $(11,12)$, The therapeutic potential of local autologous CD34+ cell therapy has been explored in clinical trials for ischemic and non-ischemic cardiomyopathy showing safety and potential efficacy. Autologous or allogenic MSCs are being explored for various systemic conditions in multiple early phase clinical trials registered on www.clinicaltrials.gov.

These BMSCs have been explored as treatment for retinal degeneration in preclinical and early clinical studies. In murine models of retinal degeneration, hematopoietic stem cells and MSCs from murine bone marrow have been shown to have protective effects on degenerating retina following intravitreal or subretinal administration $(13,14)$. Although intravitreal injection of human CD34+ BMSC was found to have no long-term ocular or systemic adverse effects in a NOD-SCID murine model of retinal ischemiareperfusion, intravitreal injection of MSCs was associated with abnormal proliferation of the cells in the eye, raising safety concerns $(12,15)$.

Since MSCs have paracrine effects on damaged tissue primarily via secretion of exosomes loaded with trophic factors, the use of exosomes from MSCs may be a safer non-cellular approach to treating retinal disease (12). Exosomes are small $(50-150 \mathrm{~nm})$ extracellular vesicles with bilipid membranes that constitute an important mechanism in cell-to-cell signaling pathways $(16,17)$. A method of harvesting a large amount of exosomes from human MSCs has been developed by culturing the cells under hypoxic, serum-free conditions (18). In an oxygen-induced retinopathy model of retinal ischemia, the intravitreal administration of exosomes derived from human MSCs was well-tolerated and showed a protective effect on retinal vasculature and retinal neurons (19).

The effects of human CD34+ BMSCs and exosomes harvested from human MSCs on degenerating retina has not been well characterized to date. In a rd1 murine model of rapid onset hereditary retinal degeneration, intravitreal injection of human CD34+ BMSCs was associated with dramatic molecular changes in the degenerating retina when chronic systemic immunosuppression was administered to avoid rejection of human cells (20). The main molecular pathways affected by CD34+ cell injection were pathways regulating apoptosis, photoreceptor maintenance and transduction. However, no protective effects on retinal function or photoreceptor morphology could be observed likely due to the rapid rate of retinal degeneration in $\mathrm{rd} 1$ mice. However, other researchers have noted that subretinal administration of murine bone marrow-derived hematopoietic stem cells may have a more dramatic protective effects on degenerating outer retina than intravitreal cell injection (14).

The purpose of our current study was to investigate the effects of human CD34+ cells on degenerating retina using an animal model with much slower rate of retinal degeneration than $\mathrm{rd} 1$ mice. Royal College of Surgeons (RCS) rats was selected since it is a commonly used animal model for evaluating the protective effects of therapy on retinal degeneration (21). In order to optimize the regenerative potential of CD34+ BMSCs, we evaluated subretinal administration of human CD34+ BMSCs alone and in combination with exosomes harvested from human MSCs. The results were compared to intravitreal administration. We present the following article in accordance with the ARRIVE reporting checklist (available at https://dx.doi.org/10.21037/atm20-4662). 


\section{Methods}

Experiments were performed under a project license (No. 19-0057-S1A0) granted by the Institutional Animal Care and Use Committee of the Seoul National University Hospital Biomedical Research Institute. Animals were treated in accordance with the Association for Research in Vision and Ophthalmology statement for the Use of Animals in Ophthalmic and Vision Research. RCS rats, 3 weeks of age at the time of injection, were used in this study. They were raised in a temperature-controlled facility with a 12-h light/dark cycle in rat cages and fed with standard rat chow. Animals were treated in accordance with the Association for Research in Vision and Ophthalmology statement for the Use of Animals in Ophthalmic and Vision Research.

\section{CD34+ cell isolation EGFP labeling}

Fresh bone marrow from a healthy human donor was obtained from Stem Express (Placerville, CA), and CD34+ cells were isolated from bone marrow as previously described (22). Briefly, fresh bone marrow aspirate was diluted 1:1 with PBS and subjected to Ficoll centrifugation. The buffy coat was aspirated to collect mononuclear cells, diluted, and washed with PBS, and centrifuged again to pellet the mononuclear cells. The cell pellet was then resuspended in PBS, and CD34+ cells were isolated from the mononuclear cell fraction by magnetic cell sorting using the Miltenyi human CD34 MicroBead Kit (Miltenyi Biotec, Auburn, CA) in accordance with the manufacturer's instructions. To label the isolated CD34+ cells with enhanced green fluorescent protein (EGFP), isolated CD34+ cells were cultured overnight at $37^{\circ} \mathrm{C} / 5 \%$ $\mathrm{CO}_{2}$ in a $35-\mathrm{mm}$ dish in HSC Proliferation Medium (23). The next day, the cells were transduced at multiplicity of infection 20 with a lentiviral vector containing EGFP and luciferase (20). The cells were resuspended at 50,000 cells $/ 4 \mu \mathrm{L}$ in PBS for the injection of CD34+ cells alone or in the exosome solution for injection of CD34+ cells in combination with exosomes.

\section{Exosome preparation}

Human bone marrow aspirates from young non-smoking males were obtained commercially (Lonza, Allendale, NJ). Human MSCs were isolated as previously described (18), and those from passage 6 were used for exosome isolation.
Exosomes were isolated as previously described (19). Briefly, MSCs were cultured in exosome isolation media (OptiMEM, Life Technologies, CA) under 1\% oxygen tension for 48 hours. The conditioned media were cleared of cells and cell debris via serial centrifugation of supernatants $(500 \mathrm{~g}$ for $10 \mathrm{~min}$ and 2,000 $\mathrm{g}$ for $15 \mathrm{~min}$, respectively). Exosomes were concentrated and washed from the resulting supernatants via tangential flow filtration with a $100-\mathrm{kDa}$ molecular weight cut-off polyethersulfone membrane (Pall, Port Washington, NY) and diafiltration wash with sterile PBS. The resulting solution was further concentrated by using a VivaSpin filtration column with a polyethersulfone membrane. The resulting concentrated exosome solution $(2.5 \mu \mathrm{g} / \mu \mathrm{L})$ was aliquoted and stored at $-80{ }^{\circ} \mathrm{C}$ until use.

\section{Subretinal and intravitreal injections}

Injections were administered in right eye of 3-week-old RCS rats. Before injection, the rats were anesthetized by an intraperitoneal injection of a mixture of tiletamine/ zolazepam and xylazine hydrochloride. The pupil was dilated with an eye drop (tropicamide $0.5 \%$ and phenylephrine hydrochloride $0.5 \%$ ), and the cornea was anesthetized with an eye drop of proparacaine hydrochloride $0.5 \%$. The cell suspension was transplanted into the subretinal space as described previously (24). A small puncture was made slightly posterior to the limbus inferiorly by using a 30G needle, and a 33-G needle connected to a $10-\mu \mathrm{L}$ microsyringe was inserted through the puncture. The needle was approached at approximately 45 degrees against the iris plane into the subretinal space until mild resistance was felt, and $4 \mu \mathrm{L}$ of the cell suspension was injected gently. With this manual injection, a subretinal bleb was made at the superior region of retina (24). For intravitreal injection, the cell suspension was injected when the needle tip was located within the vitreous cavity.

The rats were randomly assigned to one of four subretinal injection groups or three intravitreal groups: subretinal CD34+ cells (50,000 cells) alone, subretinal CD34+ cells with exosomes $(50,000$ cells $+10 \mu \mathrm{g})$, subretinal exosomes $(10 \mu \mathrm{g})$ alone, subretinal PBS, intravitreal CD34+ cells (50,000 cells) alone, intravitreal CD34+ cells with exosomes (50,000 cells+ $10 \mu \mathrm{g})$, and intravitreal exosome alone $(10 \mu \mathrm{g})$. Immediately after injection, the fundus was examined and any rats with massive subretinal or vitreous hemorrhage were excluded from the study. All animals were maintained on cyclosporin A (210 mg/L, Cipol-N; Chong Kun Dang, Seoul, Korea) 
Table 1 Summary of animals and treatment

\begin{tabular}{|c|c|c|c|c|c|c|c|c|c|}
\hline Route & Treatment (OD) & Treatment (OS) & $\begin{array}{c}\text { Number of } \\
\text { animals }\end{array}$ & \multicolumn{3}{|c|}{ Histology (sacrificed) } & \multicolumn{3}{|c|}{ ERG } \\
\hline \multirow[t]{3}{*}{ Subretinal injection } & CD34+/exosome & Control & 7 & 1 & 5 & 1 & 7 & 6 & 1 \\
\hline & CD34+ & Control & 5 & - & 5 & - & 5 & 5 & - \\
\hline & Exosome & Control & 6 & - & 4 & 2 & 6 & 6 & 2 \\
\hline \multirow[t]{3}{*}{ Intravitreal injection } & CD34+/exosome & Control & 7 & 1 & 5 & 1 & 7 & 6 & 1 \\
\hline & CD34+ & Control & 4 & - & 4 & - & 4 & 4 & - \\
\hline & Exosome & Control & 6 & - & 4 & 2 & 6 & 6 & 2 \\
\hline
\end{tabular}

administered in the drinking water from one day before injection until they were sacrificed.

\section{Electroretinography examination}

Electroretinogram (ERG) measurements were performed in both eyes before and at 2, 4, and 7 weeks after injection for all available animals at each time point using Handheld Multispecies electroretinogram unit (HMsERG system; Ocuscience LLC, Henderson, NV) as described previously (25). A scotopic flash series was recorded with a light flash intensity of $10 \mathrm{~cd} \cdot \mathrm{s} / \mathrm{m}^{2}$, and b-wave amplitude was analyzed as a parameter to reflect retinal function.

\section{Retinal histology examination}

The animals were sacrificed at 2, 4, and 7 weeks after injection. The enucleated eyes were fixed in $4 \%$ paraformaldehyde solution for $48 \mathrm{~h}$ at $4{ }^{\circ} \mathrm{C}$ and embedded in paraffin. To orient the eye in paraffin, a 10-0 nylon marking suture was placed through the conjunctiva at the superior region of each eye. The sagittal sections were cut using a microtome at $4 \mu \mathrm{m}$, placed on microscope slides, and dried overnight at room temperature. The sections were deparaffinized and subjected to standard staining with hematoxylin and eosin. The number of nuclei located in the outer nuclear layer (ONL) were counted along a 50$\mu \mathrm{m}$ length of the retina at $400-\mu \mathrm{m}$ intervals from the optic nerve within the sagittal section.

\section{Immunobistochemical examination}

For immunofluorescence analysis, paraffin sections were deparaffinized and then processed in $10 \mathrm{mM}$ citrate buffer (pH 6.0) at $95^{\circ} \mathrm{C}$ for $20-30 \mathrm{~min}$, permeabilized with $0.3 \%$ PBS-Tween for $20 \mathrm{~min}$, and then blocked with $3 \%$ BSA for 1 hour. The samples were then incubated at $4{ }^{\circ} \mathrm{C}$ overnight with the appropriate primary antibodies. The primary antibodies used were anti-MHC class I + HLA A + HLA B (Abcam) to identify human cells and antiGFAP (Abcam). The samples were washed with PBS, then incubated with Alexa Fluor 488-conjugated donkey antirabbit (Invitrogen) and Alexa Fluor 594-conjugated donkey anti-goat (Invitrogen) secondary antibodies for 1 hour at room temperature. The specimens were then treated with DAPI (Sigma) to label the nuclei, and were imaged using a confocal microscope (Leica STED CW). Presence of EGFP-labeled cell was also evaluated by the confocal microscope, using 488-nm bandpass excitation filter and 515-nm long pass emission filter.

\section{Statistical analysis}

A two-tailed Student $\mathrm{t}$ test was used to compare means. A P value $<0.05$ was considered statistically significant.

\section{Results}

The number of animals and the follow-up for each treatment group is summarized in the Table 1. Injections were performed in the right eyes only. ERG testing was performed at 2, 4, and 7 weeks after injection. The animals were sacrificed for histologic and immunohistochemical analyses, mainly at 4 weeks after injection. In some treatment groups, one or two animals were sacrificed at 2 or 7 weeks after injection to investigate the effects of treatment 
A

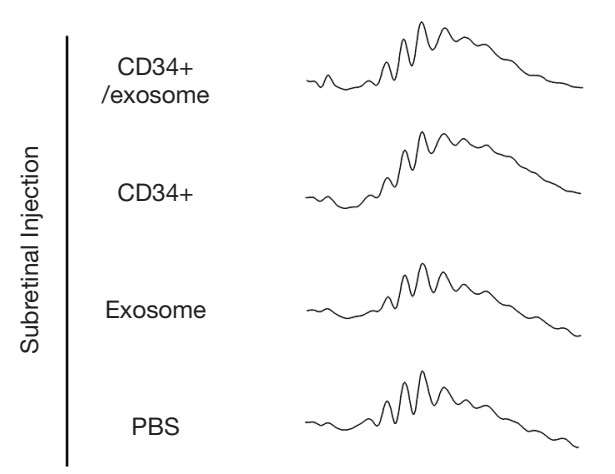

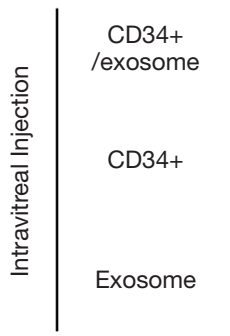

Untreated

control
Before Injection

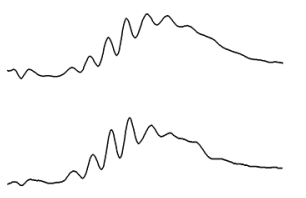

2 weeks
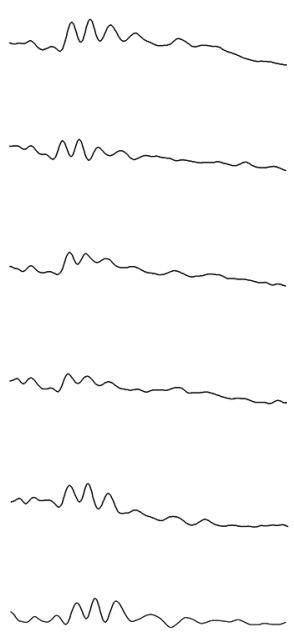

B

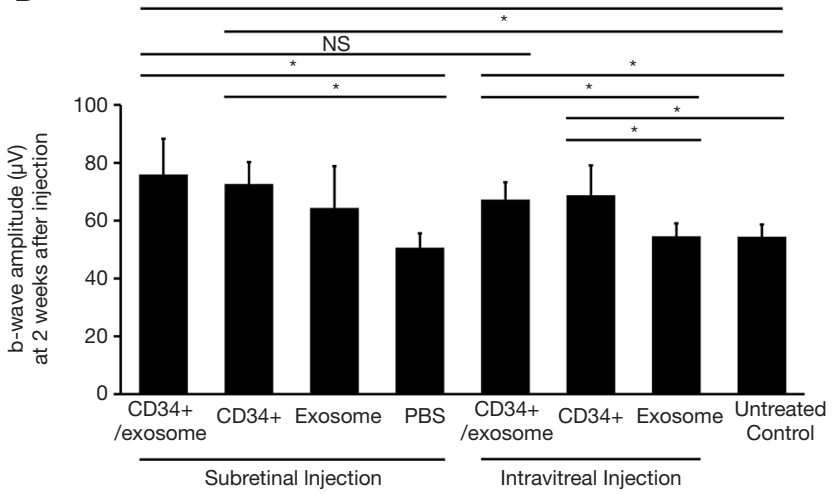

4 weeks

7 weeks
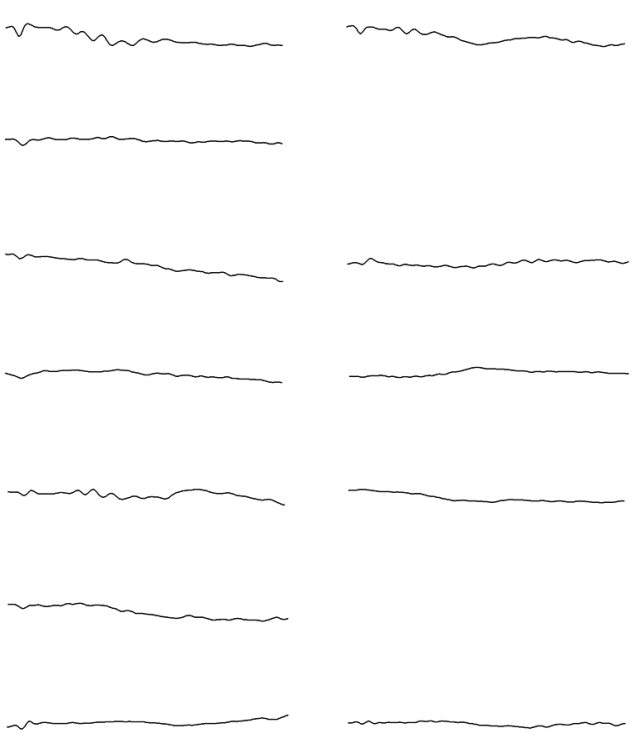

C
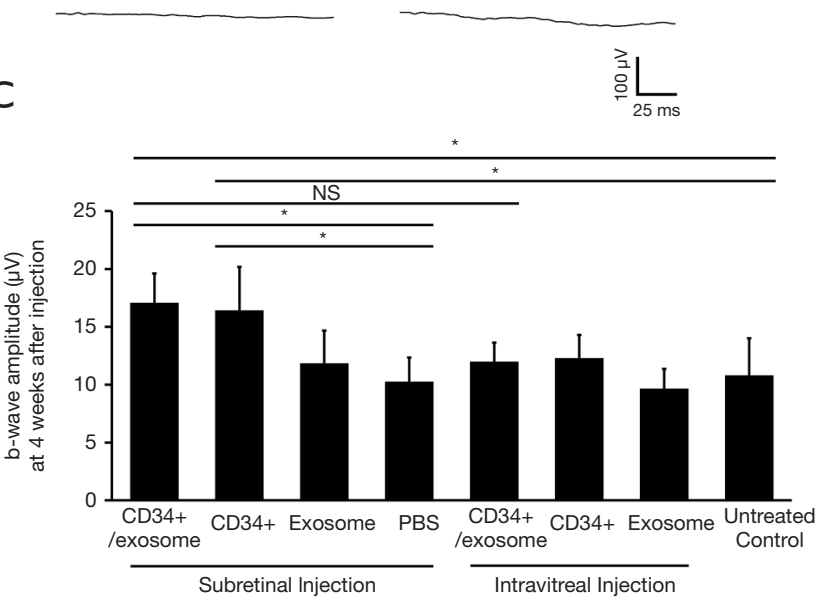

Figure 1 ERG results in Royal College of Surgeons rats following intravitreal or subretinal administration of human CD34+ cells alone or in combination with exosomes harvested from cultured human mesenchymal stem cells. (A) Representative scotopic ERG waveforms before and at 2, 4, and 7 weeks after injection in each treatment group. (B) Amplitude of ERG b-wave at 2 weeks after injection. (C) Amplitude of ERG b-wave at 4 weeks after injection. *, $\mathrm{P}<0.05$.

at other time points; however, these results were not included in the statistical analysis owing to the small sample size. All animals were in healthy condition prior to injection and euthanasia.

\section{Electroretinography testing}

At 2 weeks after injection, b-wave amplitude in the eyes that received subretinal CD34+ cells alone or combined with exosomes was significantly greater compared to eyes that received subretinal PBS or untreated contralateral eyes (Figure $1 A, 1 B$ ). There was no difference in the b-wave amplitude between eyes that received subretinal CD34+ cells alone and eyes that received subretinal CD34+ cells combined with exosomes.

The eyes that received intravitreal CD34+ cells alone 
or combined with exosomes showed significantly greater b-wave amplitude than the eyes that received intravitreal exosomes alone or untreated contralateral eyes. There was no difference in b-wave amplitude between the eyes received subretinal versus intravitreal injection of CD34+ cells with exosomes. There was no difference in the b-wave amplitude between eyes that received intravitreal CD34+ cells alone and eyes that received intravitreal CD34+ cells combined with exosomes, and between eyes that received intravitreal exosome alone and untreated contralateral eyes.

The ERG b-wave signal tended to decrease between 2 and 4 weeks after transplantation in all treatment groups. At 4 weeks after injection, the eyes that received subretinal CD34+ cells with exosomes and CD34+ cell alone had b-wave amplitude which was partially preserved and higher than that observed in eyes that received subretinal PBS or untreated contralateral eyes (Figure 1C). However, the eyes that received with intravitreal CD34+ cells with exosomes and CD34+ cell alone showed no difference when compared to the untreated contralateral eyes.

The ERG testing, performed at 7 weeks after injection showed nearly flat signals in all treatment groups. Although statistical analysis was not performed at this time point due to the small sample size, no observable differences in the ERG signal was noted among the groups.

\section{Histologic analysis}

At 4 weeks following injection, eyes that received subretinal CD34+ cells with or without exosomes showed greater preservation of ONL in the superior retina where injection into the subretinal space was performed when compared to eyes that received subretinal exosome injection alone or eyes injected with subretinal PBS (Figure 2A). The ONL nuclei counts of the superior retina was significantly greater in the eyes that received subretinal CD34+ cells when compared to eyes that received subretinal exosome alone or PBS (Figure 2B). There was no significant difference in the ONL nuclei count between eyes that received subretinal CD34+ cells alone when compared to subretinal CD34+ cells combined with exosomes. Similarly, eyes that received subretinal exosome alone showed no difference in ONL nuclei count from the subretinal PBS injected group.

In eyes that received intravitreal injection of CD34+ cells alone, CD34+ cells in combination with exosomes, or exosomes alone showed no difference in ONL nuclei count compared with the untreated contralateral eyes at 4 weeks after injection (Figure 2C). When the ONL nuclei count for the superior retina was compared between the eyes treated with subretinal versus intravitreal injection of CD34+ cells with exosomes, the subretinal group revealed significantly greater nuclei count at $0.8,1.2,1.6,2.0,2.4$ and $2.8 \mathrm{~mm}$ from the optic disc but not at other points.

\section{Immunobistochemical analysis}

To assess the survival of the transplanted CD34+ cells, immunohistochemical analysis was performed. In an eye that received subretinal CD34+ cells with exosomes, cells with annular MHC-1 staining and EGFP labeling was detected within the ONL near the injection site at 2 weeks after injection (Figure $3 A$ ). In an eye that received intravitreal CD34+ cells with exosomes, a cell with a similar staining pattern was detected on the inner side of the inner nuclear layer (Figure 3B). When the same immunohistochemical study was performed in animals sacrificed at 4 weeks after injection, a human cell was detected on the outer side of the ONL in an eye that received subretinal CD34+ cells with exosomes, but this cell was not EGFP-labeled (Figure 3C). No human cells were detected in the retina of eyes injected with PBS; only background staining was present (Figure 3D).

To assess the glial response in the degenerating retina, we performed immunostaining for GFAP, an indicator of Müller glial reactivity. At 4 weeks after treatment, eyes injected with subretinal PBS or untreated contralateral eyes showed increased GFAP staining with the extension of GFAP-labeled Müller cell processes into the subretinal space (Figure 4). In eyes injected with intravitreal CD34+ cells and exosomes, increased GFAP staining was also observed in the subretinal space. In contrast, eyes that received subretinal CD34+ cells alone or combined with exosomes showed no staining of GFAP in the subretinal space, indicating no increased glial stress due to degeneration. At 7 weeks after injection, GFAP staining in the retina of eyes that received subretinal CD34+ cells were increased compared with 4 weeks after injection; however, there was still no staining in the subretinal space.

\section{Discussion}

This study demonstrates that both subretinal and intravitreal injection of human CD34+ BMSCs can provide functional rescue of degenerating retina in RCS rats, suggesting their potential protective effects. Human CD34+ BMSCs are a good candidate for cell therapy because they can be obtained relatively easily from bone 
A

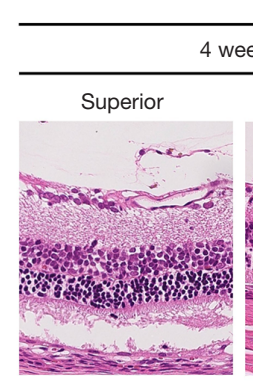

-

CD34+

BS

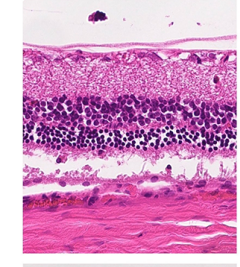

Exosome

B

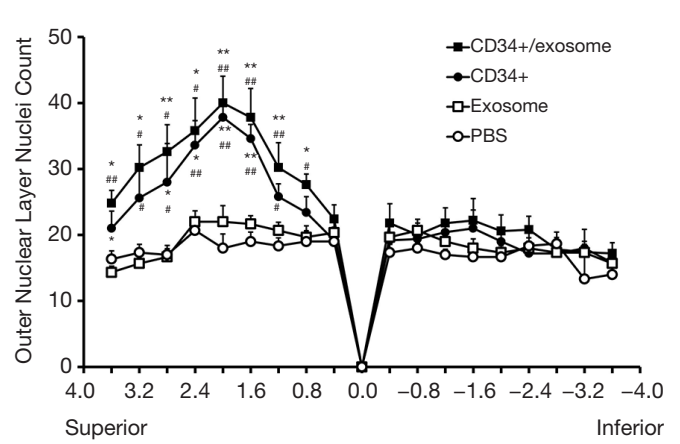

Subretinal Injection

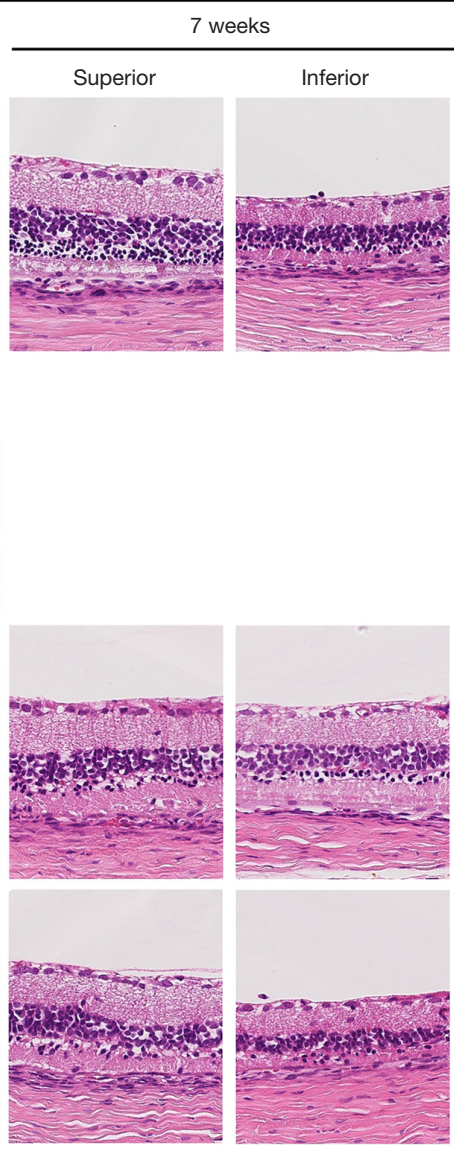

C

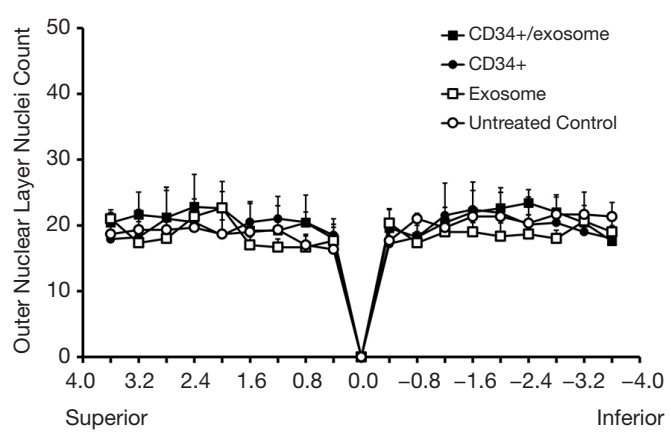

Figure 2 Histological analysis of the retina of Royal College of Surgeons rats at 4 and 7 weeks after intravitreal or subretinal injection of human CD34+ cells, alone or in combination with exosomes harvested from cultured human mesenchymal stem cells. (A) Eyes treated with subretinal injection of CD34+ cells with or without exosomes showed more preserved ONL in the superior retina sectioned $2 \mathrm{~mm}$ from the optic nerve head at 4 weeks after injection when compared to those treated with exosomes alone or PBS. This effect appears attenuated at 7 weeks after injection. In the sections of the inferior retina, degenerative changes in the ONL were similar among the treatment groups. Eyes that received intravitreal injection of human CD34+ cells with and without exosomes or exosomes alone showed less preserved ONL in the superior retina when compared to eyes treated with subretinal injection of CD34+ cells. (B) The number of ONL nuclei at 4 weeks after subretinal injection counted along a $50-\mu \mathrm{m}$ length of retina from superior to inferior through the optic nerve ${ }^{*}, \mathrm{P}<0.05,{ }^{* *}, \mathrm{P}<0.01$ versus

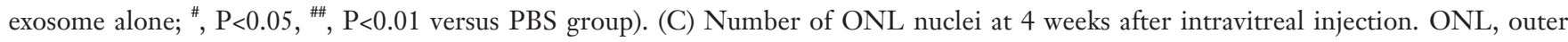
nuclear layer. 


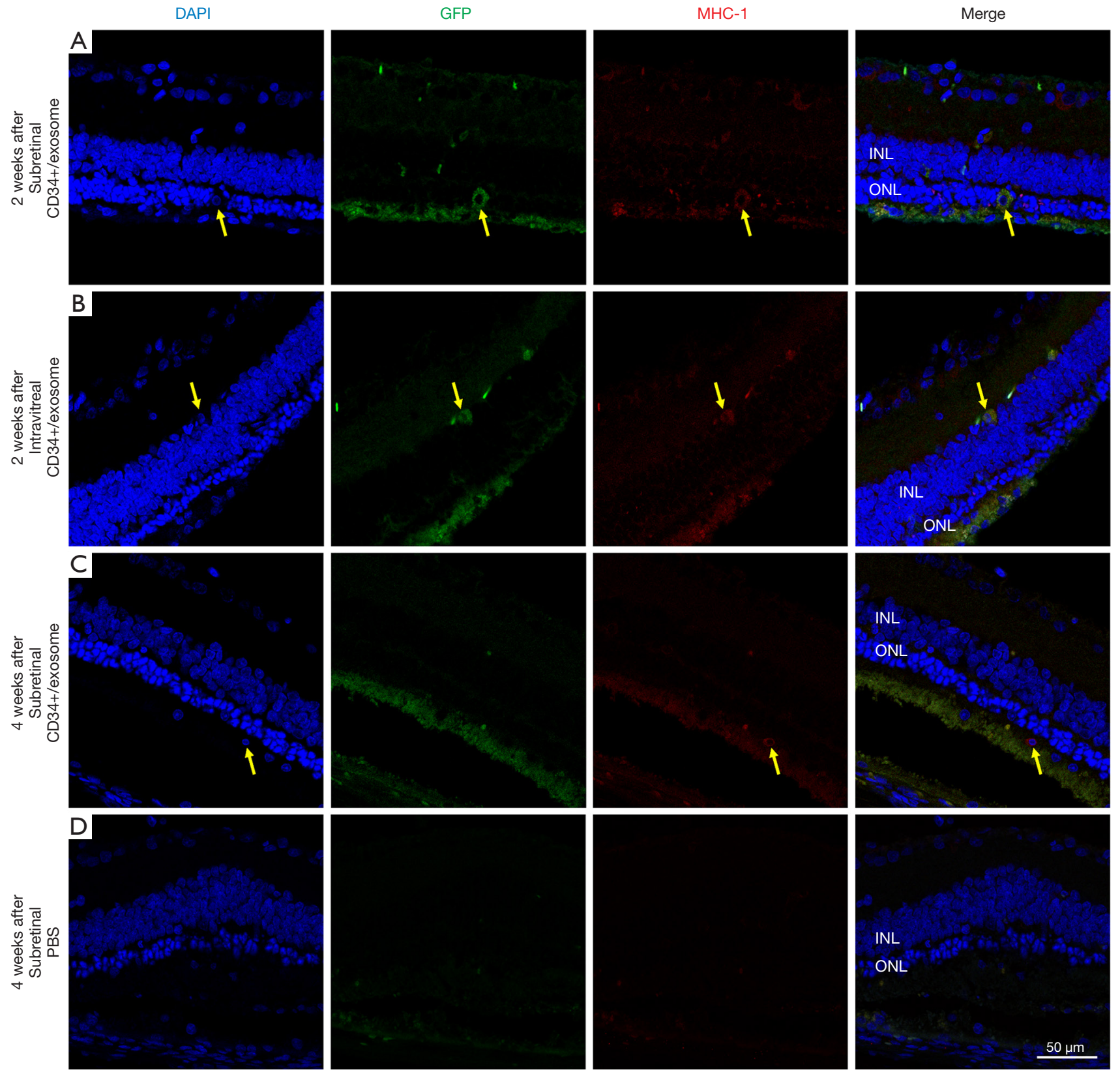

Figure 3 Immunohistochemical analysis at 2 and 4 weeks after injection of CD34+ cells. At 2 weeks after injection of CD34+ cells with exosomes, a cell with annular staining of human antibody (anti-MHC-1) and EGFP labeling was detected within the ONL after subretinal injection (A) and in the inner nuclear layer after intravitreal injection (B), respectively. At 4 weeks after subretinal injection of CD34+ cells with exosomes, a cell that stained for human antibody but not labeled with EGFP was detected on the outer side of ONL (C). In contrast, only minimal background fluorescence was detected in the retina of an eye treated with subretinal PBS (D). EGFP, enhanced green fluorescent protein; ONL, outer nuclear layer.

marrow and administered autologously, obviating the need for immunosuppression (12). In the current study, the RCS rat, a rodent model of retinitis pigmentosa in humans, was selected because the time course for development of retinal degeneration is much slower when compared to rd1 mouse (26). The protective effect of human CD34+ 

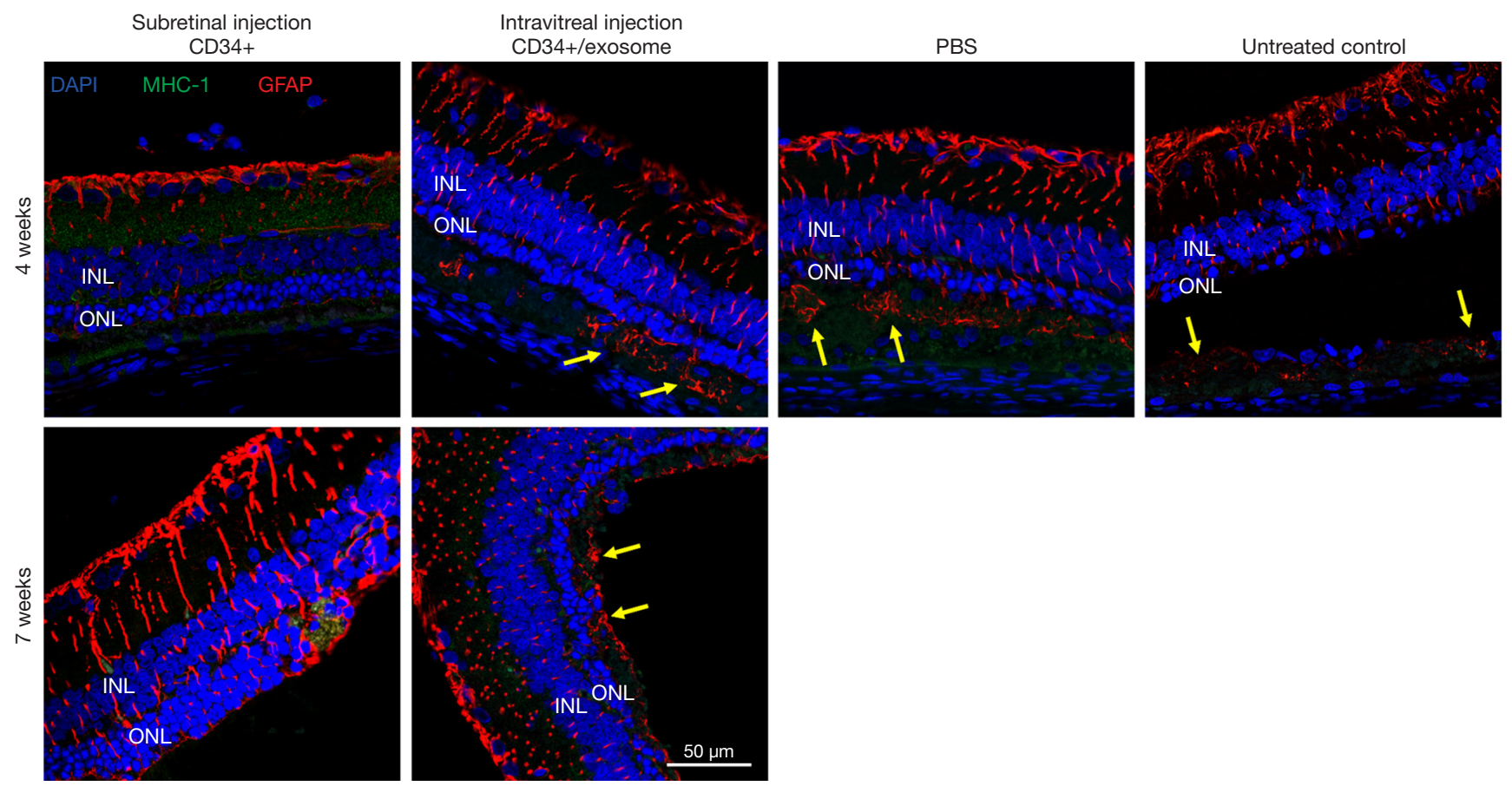

Figure 4 Immunohistochemical analysis to evaluate glial response of the retina in Royal College of Surgeons rats following intravitreal and subretinal injection of human CD34+ cells. After 4 weeks, eyes that had subretinal injection of PBS or intravitreal injection of CD34+ cells showed greater GFAP staining with extension of GFAP-stained Müller cell processes into the subretinal space when compared to eyes that received subretinal CD34+ cells injection. At 7 weeks, eyes that had subretinal injection of CD34+ cells had increased GFAP staining within the retina that did not extend into the subretinal space.

BMSCs on retinal function or morphology may be difficult to demonstrate in a murine model of rapidly progressive retinal degeneration such as rd1 mice (20).

In this study, CD34+ cells and exosomes from MSCs were transplanted in RCS rats at 3 weeks of age, which is when retinal degeneration starts (27). Both subretinal and intravitreal CD34+ cells showed functional rescue effects at 2 weeks after transplantation via ERG, but these effects were attenuated by 4 weeks after transplantation. Only the groups with subretinal CD34+ cells, alone or in combination with exosomes, had a low but detectable b-wave at 4 weeks such that a significant difference could be noted when compared to PBS injected or contralateral untreated eyes. However, by 7 weeks after injection, all eyes had flat ERG signal. Subretinal injection of exosomes from MSCs had no significant effect on ERG at all time points. The histologic findings at 4 weeks after injection were consistent with the ERG results. Subretinal injection of CD34+ cells resulted in a regional preservation of ONL nuclei around the injection site when compared with PBS injected or contralateral untreated eyes. However, subretinal injection of exosomes derived from human MSCs had no observable effects on retinal degeneration based on histology, alone or in combination with subretinal CD34+ cells.

The protective effects of CD34+ cells appear to wane over time suggesting a possible transient effect. The rescue effects based on ERG and histology were attenuated and not observed by 7 weeks after transplantation. However, it should be noted that we administered adult human CD34+ cells into rat eyes, subjecting these cells to immune rejection. Only cyclosporine was used as immunosuppressive therapy for the study duration and immunohistochemistry shows fewer and fewer human cells in the retina with longer study follow-up. This observation raises concerns that the human CD34+ cells are not surviving long-term in these rat eyes. Although a previous study used cyclosporine in RCS rats for systemic immunosuppression to evaluate the effect of intravitreal and subretinal human MSCs (21), MSCs may not be subject to immune rejection as much as adult human CD34+ cells. In prior studies evaluating the effect of human 
CD34+ cells in mice beyond a few days, NOD-SCID mice or continuous subcutaneous infusion with tacrolimus and rifamycin was used to avoid rejection of human cells $(15,20,28)$. In these studies, long-term incorporation of human cells in the retina could be observed.

In this study, eyes treated with subretinal CD34+ cells showed significantly greater nuclei counts in the ONL at the superior retina near the subretinal injection site at 4 weeks after injection, whereas those treated with intravitreal CD34 cells showed comparable number of nuclei to untreated contralateral control eyes. The observation parallels the finding of a recent study in RCS rats showing a more pronounced and longer lasting rescue effect following subretinal injection of human MSCs (21). It can be hypothesized that the paracrine trophic effect of CD34+ cells may be greater when the cells were placed closer to the damaged cells in the outer retina, i.e., photoreceptor and RPE cells presumably due to limited migration of the cells or secreted factors. In a murine model of retinal degeneration, it was noted that subretinal administration of murine hematopoietic stem cells resulted in photoreceptor regeneration from fusion of injected cells with Müller cells in vivo to develop photoreceptor progenitor cells (14). This effect was not observed after intravitreal cell injection. Although it is unknown if such cellular fusion can occur with human CD34+ cells, these observations suggest that intravitreal injection of CD34+ cells could have a more limited benefit on photoreceptor survival in retinal degenerative conditions. In contrast, protective effect of intravitreal injection of human CD34+ cells have been shown in murine models of retinal vasculopathy with longterm incorporation of the human cells in the inner retina $(15,28)$, and this suggests intravitreal administration of CD34+ cells may be the preferred route for inner retinal disorders.

On the other hand, the preservation of ONL nuclei observed after subretinal injection of CD34+ cells were most pronounced near the injection site in the superior retina. A similar pattern of ONL preservation in the vicinity of the injection site was reported after subretinal treatment with microRNA in RCS rats (29). These findings suggest that the regenerative effects of subretinal CD34+ cells or other therapeutic agents may be limited in its lateral spread. Thus, subretinal administration may be ideal for eyes with focal outer retinal degeneration, such as macular degeneration. Indeed, clinical trials exploring cell therapy for macular degeneration often use subretinal route of cell administration $(4,8,9)$.
Subretinal or intravitreal injection of exosomes harvested from human MSCs showed no observable beneficial effect on degenerating retina when injected alone or in combination with CD34+ cells. In contrast, exosomes derived from MSCs using the same protocol as in this study have been shown to have protective effects on the retina and retinal vasculature in a murine model of retinal ischemia (19). The discrepancy in the findings between these two studies may be related to differences in the disease models being studied. Since the exosomes were harvested from MSCs under hypoxic conditions, their content may consist mainly of pro-angiogenic paracrine effectors which are likely to be more beneficial in eyes with retinal vascular disease (18). Detailed proteomic analysis of exosomes harvested from MSCs using the protocol used in this study showed 1,927 different proteins, including various growth factors and NF- $\mathrm{NB}$ signal pathway proteins.

This study has some limitations. First, CD34+ cells are a heterogeneous group of cells including endothelial progenitor cells. Thus, it is possible that the effector cells in eyes with retinal degeneration may differ from those cells that respond to retinal ischemia. Therefore, further investigation is required to elucidate the mechanisms of action of CD34+ cells in different retinal disorders. Second, we had a limited number of animals per treatment arm. Thus, our study may not be powered to detect more subtle effects of the CD34+ cells or exosomes on the degenerating retina. Third, due to a limited number of animals available for our study, a control group of intravitreal PBS was not included. Fourth, the ERG recordings in our study was limited to RCS rats at various ages. Although ERG of wildtype animal as a control was not assessed in this study, ERG amplitude of wild-type animal has been noted to be comparable to RCS rat at the age of 3 weeks and to remain stable thereafter $(26,27)$. Thus, ERG traces of RCS rats performed just before injection at the age of 3 weeks were included in Figure 1.

\section{Conclusions}

In summary, our study showed that both subretinal and intravitreal injections of CD34+ BMSCs were well tolerated and exerted functional rescue effects on retinal degeneration in RCS rats. The administration of exosome derived from human MSCs under hypoxic conditions showed no effect on retinal degeneration when administered alone or in combination with CD34+ cells. Further investigations are needed to improve our understanding of the mechanism 
of action of CD34+ cells in rescuing eyes with retinal degeneration. Such studies would allow development and design of the ideal retinal regenerative therapy using human CD34+ cells.

\section{Acknowledgments}

Funding: This study was partially supported by the grant of the Korea Health Technology R\&D Project of the Korea Health Industry Development Institute funded by the Korean Ministry of Health and Welfare (grant number: HI14C1277). This work was also partially supported by the Korean Association of Retinal Degeneration. This work was also supported by the National Center for Advancing Translational Sciences and the National Institutes of Health (grant numbers UL1 TR001860 and linked award KL2 TR001859, and T32-HL086350). All funders did not participate in the design of the study, conducting the study, data management, data analysis, interpretation of the data, and the preparation, review, and approval of the manuscript.

\section{Footnote}

Provenance and Peer Review: This article was commissioned by the editorial office, Annals of Translational Medicine for the series "Novel Tools and Therapies for Ocular Regeneration". The article has undergone external peer review.

Reporting Checklist: The authors have completed the ARRIVE reporting checklist. Available at https://dx.doi. org/10.21037/atm-20-4662

Data Sharing Statement: Available at https://dx.doi. org/10.21037/atm-20-4662

Peer Review File: Available at https://dx.doi.org/10.21037/ atm-20-4662

Conflicts of Interest: All authors have completed the ICMJE uniform disclosure form (available at https://dx.doi. org/10.21037/atm-20-4662). The series "Novel Tools and Therapies for Ocular Regeneration" was commissioned by the editorial office without any funding or sponsorship. SSP served as the unpaid Guest Editor of the series and serves as an unpaid editorial board member of Annals of Translational Medicine from Oct 2019 to Sep 2021, and reports grants from Allergan, grants from Roche/Novartis, outside the submitted work. JDA reports that he has a patent pending associated with exosome technology. The authors have no other conflicts of interest to declare.

Ethical Statement: The authors are accountable for all aspects of the work in ensuring that questions related to the accuracy or integrity of any part of the work are appropriately investigated and resolved. Experiments were performed under a project license (No. 19-0057S1A0) granted by the Institutional Animal Care and Use Committee of the Seoul National University Hospital Biomedical Research Institute. Animals were treated in accordance with the Association for Research in Vision and Ophthalmology statement for the Use of Animals in Ophthalmic and Vision Research.

Open Access Statement: This is an Open Access article distributed in accordance with the Creative Commons Attribution-NonCommercial-NoDerivs 4.0 International License (CC BY-NC-ND 4.0), which permits the noncommercial replication and distribution of the article with the strict proviso that no changes or edits are made and the original work is properly cited (including links to both the formal publication through the relevant DOI and the license). See: https://creativecommons.org/licenses/by-nc-nd/4.0/.

\section{References}

1. Verbakel SK, van Huet RAC, Boon CJF, et al. Nonsyndromic retinitis pigmentosa. Prog Retin Eye Res 2018;66:157-86.

2. Bressler NM. Age-Related Macular Degeneration Is the Leading Cause of Blindness. JAMA 2004;291:1900-1.

3. Pearson RA, Ali RR. Spotlight Unlocking the Potential for Endogenous Repair to Restore Sight. Neuron 2018;100:524-6.

4. Schwartz SD, Regillo CD, Lam BL, et al. Human embryonic stem cell-derived retinal pigment epithelium in patients with age-related macular degeneration and Stargardt's macular dystrophy: follow-up of two open-label phase 1/2 studies. Lancet 2015;385:509-16.

5. Mandai M, Watanabe A, Kurimoto Y, et al. Autologous induced stem-cell-derived retinal cells for macular degeneration. N Engl J Med 2017;376:1038-46.

6. Shirai H, Mandai M, Matsushita K, et al. Transplantation of human embryonic stem cell-derived retinal tissue in two primate models of retinal degeneration. Proc Natl Acad Sci U S A 2016;113:E81-90. 
7. Mandai M, Fujii M, Hashiguchi T, et al. iPSC-Derived Retina Transplants Improve Vision in rd1 EndStage Retinal-Degeneration Mice. Stem Cell Reports 2017;8:69-83.

8. Mehat MS, Sundaram V, Ripamonti C, et al. Transplantation of Human Embryonic Stem Cell-Derived Retinal Pigment Epithelial Cells in Macular Degeneration. Ophthalmology 2018;125:1765-75.

9. Singh MS, Park SS, Albini TA, et al. Retinal stem cell transplantation: Balancing safety and potential. Prog Retin Eye Res 2020;75:100779.

10. Jin ZB, Gao ML, Deng WL, et al. Stemming retinal regeneration with pluripotent stem cells. Prog Retin Eye Res 2019;69:38-56.

11. Strauer BE, Brehm M, Zeus T, et al. Repair of infarcted myocardium by autologous intracoronary mononuclear bone marrow cell transplantation in humans. Circulation 2002;106:1913-8.

12. Park SS, Moisseiev E, Bauer G, et al. Advances in bone marrow stem cell therapy for retinal dysfunction. Prog Retin Eye Res 2017;56:148-65.

13. Otani A, Dorrell MI, Kinder K, et al. Rescue of retinal degeneration by intravitreally injected adult bone marrowderived lineage-negative hematopoietic stem cells. J Clin Invest 2004;114:765-74.

14. Sanges D, Simonte G, Di Vicino U, et al. Reprogramming Müller glia via in vivo cell fusion regenerates murine photoreceptors. J Clin Invest 2016;126:3104-16.

15. Park SS, Caballero S, Bauer G, et al. Long-term effects of intravitreal injection of GMP-grade bone-marrowderived CD34 + cells in NOD-SCID mice with acute ischemia-reperfusion injury. Invest Ophthalmol Vis Sci 2012;53:986-94.

16. Théry C, Ostrowski M, Segura E. Membrane vesicles as conveyors of immune responses. Nat Rev Immunol 2009;9:581-93.

17. Lee HK, Finniss S, Cazacu S, et al. Mesenchymal stem cells deliver exogenous miRNAs to neural cells and induce their differentiation and glutamate transporter expression. Stem Cells Dev 2014;23:2851-61.

18. Anderson JD, Johansson HJ, Graham CS, et al. Comprehensive proteomic analysis of mesenchymal stem cell exosomes reveals modulation of angiogenesis via nuclear factor-kappaB signaling. Stem Cells 2016;34:601-13.

19. Moisseiev E, Anderson JD, Oltjen S, et al. Protective Effect of Intravitreal Administration of Exosomes Derived from Mesenchymal Stem Cells on Retinal Ischemia. Curr Eye
Res 2017;42:1358-67.

20. Moisseiev E, Smit-McBride Z, Oltjen S, et al. Intravitreal administration of human bone marrow CD34+ stem cells in a murine model of retinal degeneration. Invest Ophthalmol Vis Sci 2016;57:4125-35.

21. Tzameret A, Sher I, Belkin M, et al. Transplantation of human bone marrow mesenchymal stem cells as a thin subretinal layer ameliorates retinal degeneration in a rat model of retinal dystrophy. Exp Eye Res 2014;118:135-44.

22. Park SS, Bauer G, Abedi M, et al. Intravitreal autologous bone marrow cd34+ cell therapy for ischemic and degenerative retinal disorders: Preliminary phase 1 clinical trial findings. Invest Ophthalmol Vis Sci 2014;56:81-9.

23. Walker JE, Chen RX, McGee J, et al. Generation of an HIV-1-Resistant Immune System with CD34+ Hematopoietic Stem Cells Transduced with a TripleCombination Anti-HIV Lentiviral Vector. J Virol 2012;86:5719-29.

24. Park SW, Kim JH, Park WJ, et al. Limbal approachsubretinal injection of viral vectors for gene therapy in mice retinal pigment epithelium. J Vis Exp 2015;(102):e53030.

25. Ma DJ, Lim MS, Park UC, et al. Magnetic iron oxide nanoparticle labeling of photoreceptor precursors for magnetic resonance imaging. Tissue Eng Part C Methods 2019;25:532-42.

26. Rösch S, Aretzweiler C, Müller F, et al. Evaluation of Retinal Function and Morphology of the Pink-Eyed Royal College of Surgeons (RCS) Rat: A Comparative Study of in Vivo and in Vitro Methods. Curr Eye Res 2017;42:273-81.

27. Adachi K, Takahashi S, Yamauchi K, et al. Optical coherence tomography of retinal degeneration in royal college of surgeons rats and its correlation with morphology and electroretinography. PLoS One 2016;11:e0162835.

28. Yazdanyar A, Zhang P, Dolf C, et al. Effects of intravitreal injection of human CD34+ bone marrow stem cells in a murine model of diabetic retinopathy. Exp Eye Res 2020;190:107865.

29. Lian C, Lou H, Zhang J, et al. MicroRNA-24 protects retina from degeneration in rats by down-regulating chitinase-3-like protein 1. Exp Eye Res 2019;188:107791.

Cite this article as: Park UC, Park SS, Kim BH, Park SW, Kim YJ, Cary W, Anderson JD, Nolta JA, Yu HG. Subretinal versus intravitreal administration of human CD34+ bone marrow-derived stem cells in a rat model of inherited retinal degeneration. Ann Transl Med 2021;9(15):1275. doi: 10.21037/ atm-20-4662 